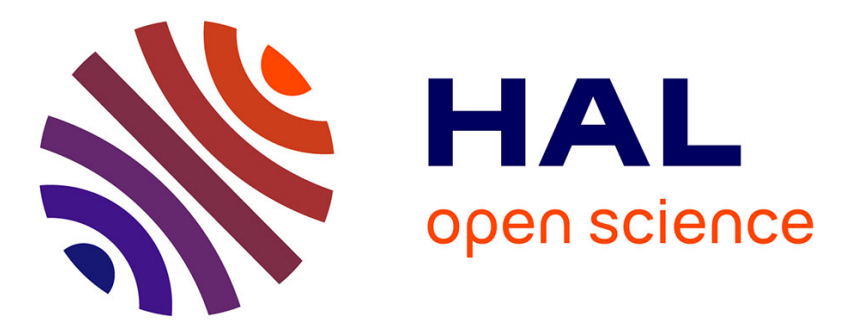

\title{
What Characterizes a Good Software Tester? - A Survey in Four Norwegian Companies
}

\author{
Anca Deak
}

\section{To cite this version:}

Anca Deak. What Characterizes a Good Software Tester? - A Survey in Four Norwegian Companies. 26th IFIP International Conference on Testing Software and Systems (ICTSS), Sep 2014, Madrid, Spain. pp.161-172, 10.1007/978-3-662-44857-1_11 . hal-01405283

\section{HAL Id: hal-01405283 \\ https://hal.inria.fr/hal-01405283}

Submitted on 29 Nov 2016

HAL is a multi-disciplinary open access archive for the deposit and dissemination of scientific research documents, whether they are published or not. The documents may come from teaching and research institutions in France or abroad, or from public or private research centers.
L'archive ouverte pluridisciplinaire HAL, est destinée au dépôt et à la diffusion de documents scientifiques de niveau recherche, publiés ou non, émanant des établissements d'enseignement et de recherche français ou étrangers, des laboratoires publics ou privés. 


\title{
What characterizes a good software tester? - a survey in four Norwegian companies
}

\author{
Anca Deak \\ Department of Computer Science \\ Norwegian University of Science and Technology (NTNU) \\ Trondheim, Norway \\ deak@idi.ntnu.no
}

\begin{abstract}
The aim of this paper is to identify the most favourable perceived characteristics of software testers and contrast them with the group of existing software engineers' characteristics provided by prior research. We conducted a qualitative survey among four software development companies using interviews as a main method for data collection and the thematic-analysis approach for analyzing the data. Our findings describe the characteristics which are perceived as most valuable for a tester based on the working experience accumulated by the participant testers and testing managers. The study findings suggest that there are differences between the characteristics of a software engineer and a software tester, with communication and possessing an IT background being cited as the most frequently favoured characteristics for ensuring a high performing tester.
\end{abstract}

Keywords: software testers, software testing, software engineers, characteristics, human factors

\section{Introduction}

Successful software projects outcomes are highly dependent on the skills and commitment of the testers involved. Having good testers will significantly improve the odds of project success and the delivery of high quality products. Since testing projects often occur under tight deadlines, budget constraints and organizational challenges, the success of a project can be highly dependent on the employee's characteristics.

There are not many studies focusing on the testers or on testing teams, although it is acknowledged that some of the problems associated with software testing in the industry are not technical, but originate from other sources, such as the sociotechnical environment and organizational structure of the company as presented in Rooksby, Rouncefield and Sommerville study [1]. Although there is extensive work on characteristics of software engineers, to our knowledge there is a lack of research 
focusing specifically on the characteristics of software testers. The goal of this study is to empirically determine specific traits for software testers and contrast them with software engineers' characteristics provided by prior research. A better understanding of these traits could assist in the identification of individuals with potential to be high performing testers and help the software companies within their recruiting process.

The remainder of this article is structured as follows. A literature background on characteristics of software engineers and software testers is presented in Section 2. We describe the research questions and design of the survey in Section 3. In Section 4 , we present the survey's results. Section 5 discusses the lessons learned while Section 6 summarizes the findings and suggest areas for further research.

\section{Characteristics of software engineers and software testers}

\subsection{Research on characteristics of software engineers}

Software Engineers characteristics was the scope of a systematic literature review conducted by Beecham et al. [2], in which 92 papers published between 1980 and June 2006 were analyzed. The result of this study provided 16 characteristics of the software engineer together with a group of motivators and de-motivators which were identified in the literature. The study conducted by Beecham et al. [2] indicates that several factors have influence on a software engineers' motivation. One of these factors is related to the personal characteristics of the software engineer. The most cited characteristics of software engineers were: Growth orientation, Need for independence and Low need for social interaction which is associated with introverts.

These characteristics have two set of determinants: the control factors and the moderators. The control factors group relate to individual's personality, their career paths and their competencies. The second group, the moderators, are considered external factors that can influence the characteristics, such as: career stage and culture which are most often cited, followed by the type of job and the type of organisation which have less influence. A subsequent study by Franca et al. [3] extended and updated this research by analyzing 53 papers published between March 2006 to August 2010. As a result, another three characteristics were identified: Competent in Management, Flexible and Have fear of punishment. Most of the studies involved in these two literature reviews were quantitative survey studies and they provided important insights into characterizing the factors and results related to motivation. One limitation of the two literature reviews, is that the majority of the studies are referring to the job itself as being the main motivational factor.

Since the position of software engineer can contain multiple roles and responsibilities which can vary from one position to another, more information about the characteristics which influence the motivation of software engineer is required. Our studies focus on software testers, who are often considered as software engineers in job title terminology, but may have different responsibilities than their fellow developers. 
Lack of motivation has been seen as an important factor for failure in software development projects [4] and a recent study of a vendor organization's testing show that the quality of testing is affected by the motivation of the testers [5]. Previous studies observing the testing activities examined how the organization and the relationships between the members of a software development team are impacting the testing process and implicitly the quality of the product [6]. We wanted to build on this research by determining the characteristics which influence the motivation of different types of testers'.

\subsection{Research on software testers' characteristics}

Testing literature written by practitioners proposed skills and characteristics which they considered most important for software testers. Pettichord provides a list of twelve desired traits which he compares between testers and developers [7]. Good testers are expected to have broad knowledge of many domains, learn new things quickly, focus on user needs and behaviour, think empirically, tolerate tedium, be sceptical, concentrate on reporting problems and be comfortable with conflicts. Black [8] suggests concrete desired skills, such as the ability to interpret the product requirements and other types of available material as well as the ability to write good defect reports. The skills are also described in Pol [9] study, but they are not so strongly emphasized. Both studies acknowledged the need for strong knowledge both of software development and application domain of the product.

An ethnographical study of a small start-up software product company conducted by Martin, Rooksby et al. [10] studied how testing is performed in an environments with extremely volatile requirements. Their study focused on integration and acceptance testing done in the company. The results showed that testers working in contexts where requirements where not defined in detailed and without any strict processes, needed understanding of the business and experience in the domain and techniques that were used to test the product. Testers were also required to possess good skills in test automation.

The importance of experience in testing was also studied by Beer and Ramler [11] trough a multiple-case study, which covered three industrial software projects. The results of the study identified two kinds of experience: experience in testing and experience with the domain of the product. Experience in testing was used in general management of the testing and especially in automation of the testing. Testers who have accumulated experience in the business domain and have strong business knowledge and expertise proved valuable when performing test case design, regression test case selection, or facing incomplete or evasive requirements and specifications.

A case study on high performing testers reported by [12], was conducted in three Finnish software product companies. The aim of the study was to identify the characteristics which were considered important for testers with high performance. Among the findings were that experience, reflection, motivation and personal characteristics had the highest importance. The results also strengthen the findings of 
the previous studies by stating that domain knowledge and specialized technical skills were considered more relevant that test case design and test planning skills.

Taipale and Smolander conducted a qualitative study [13], which explored the software-testing practices and suggested improvements in this process based on the knowledge acquired during their study. Their improvement proposition include adjusting testing according to the business orientation of the company, enhanced testability of software components, efficient communication, early involvement of testers and increased interaction between developers and testers. The same group of authors conducted another study, focusing on the conditions that influence software testing as an online service, looked among other at the skills required for software testing [14]. The results of the study showed that in order to assure a successful execution of an online testing process, both technical and soft skills are required. An ability to adjust to different working methods, increased understanding of customer's business needs and requirements, communication, project management, technical skills alongside with a flexibility to learn and adapt to evolving technologies were considered the required testing skills.

An empirical study of a vendor organization's testing team conducted by Shah, Harrold and Sinha [5], studied the perceptions the participants had about testing. The testers showed enthusiasm about their job and they have a positive attitude toward testing, which is the opposite of the common attitude towards testing, as being not so high valued as a software development job. A desire for innovation and a high values among the testers were also observed in the same study. In addition, the study shows that the quality of testing is affected by the motivation of the testers and emphasizes the need for appreciating testers' efforts.

\section{Research questions and method}

The aim of this paper is to study the characteristics of software testers (RQ1), and to contrast the group of characteristics derived from our study with the group of existing characteristics for software engineers provided by two previous systematic literature reviews of motivation in software engineering, [2] and [3], (RQ2). Thus, we investigated the following two research questions:

- RQ1 What are the characteristics perceived as important of software testers?

- $\quad$ RQ2 Are there specific characteristics that separates software testers from a software engineer?

The target population for our study is represented by software testing professionals. In the testers' category we will consider all software engineers who have software testing as their main job responsibility. A total of 14 participants from four companies were interviewed, with seven interviews being performed in agile working teams from two companies, while the other seven interviews were done in two companies following the traditional development methodology. The participants included testers 
and testing managers who face the daily problems of software testing activities. The companies and interviewees are described in Table 1.

Table 1: Companies and interviewees

\begin{tabular}{|l|l|l|l|l|}
\hline Business & Size & Methodology & Testing & Interviewees \\
\hline Company A & Agile, TDD & Functional & $\begin{array}{l}\text { Testing manager } \\
\text { Tester (3) }\end{array}$ \\
\hline $\begin{array}{l}\text { Software producer } \\
\text { and service provider }\end{array}$ & $\begin{array}{l}\text { medium } \\
\text { international }\end{array}$ & Agile, Scrum & $\begin{array}{l}\text { Non } \\
\text { functional }\end{array}$ & $\begin{array}{l}\text { Testing manager } \\
\text { Tester (2) }\end{array}$ \\
\hline Company B & $\begin{array}{l}\text { Software producer } \\
\text { and testing provider }\end{array}$ & $\begin{array}{l}\text { medium } \\
\text { national }\end{array}$ & Functional & $\begin{array}{l}\text { Testing manager } \\
\text { Tester (2) }\end{array}$ \\
\hline Company C & $\begin{array}{l}\text { Traditional } \\
\text { nedium }\end{array}$ & Fofional \\
\hline Software producer & $\begin{array}{l}\text { large } \\
\text { international }\end{array}$ & Traditional & Functional & $\begin{array}{l}\text { Testing manager (2) } \\
\text { Tester (2) }\end{array}$ \\
\hline Company D
\end{tabular}

During the interviews we used semi-structured questions in order to encourage the respondents to provide us with their own reflections and use their own terms. During the interviews the respondents were encouraged to freely express their opinions, by guaranteeing their anonymity and assuring them that the records will be accessible only to the researchers involved in this study. The interview guideline included both closed questions, for which responses will be easier to analyze and compare, and open questions which will allow the participants to point out issues that were not mentioned in the closed-form questions. The open questions were theme based and concerned problems of testing, collaboration within their team, relationships with fellow colleagues, plus positive and negative aspects of their daily activities. In addition, we enquired about their working environment, schedules and the influence of the business domain. The same set of questions will enable us to see if there is a difference between the characteristics of a tester and those of the more general category of software engineers.

The interviews varied between half an hour and one hour and a half, and were performed on the company premises, in quiet meeting rooms. Each participant was interviewed individually. During the interviews the respondents were encouraged to express their opinions freely and as recommended by Myers and Newman [15], we used a mirroring technique in questions and answers in order to encourage the respondents to share their stories. During the interviews we asked the participants to talk about both current events and to reflect retrospectively on previous scenarios. All interviews were recorded and transcribed, and the transcriptions were sent to each participant for final checking and approval. Notes were also taken with the leading issues for each interview. The transcribed interviews were coded in several rounds.

Starting the process of analyzing the research data available, we first identified the segments of text relevant to the two research questions and discarded those bearing no relation to it. The transcripts were reexamined several times, and the coding process was performed in repeated rounds and the results were peer reviewed and discussed. Afterwards, we proceeded with the coding phase and labelled each segment by means of one or more easily recognizable terms or categories, using a software tool designed 
for qualitative analysis (NVivo 10) in which we imported all the transcripts. Afterwards the codes were analyzed and similar codes were aggregated into more general codes in order to reduce the number of codes utilized and retrieve the emerging categories. We derived the categories using the framework provided in the studies by Beecham et al. [2] as a model for constructing a list of characteristics for software testers. Each category and code can be linked to quotations from the interviews and these are used to strengthen and enhance the results. The list combining characteristics from both studies [2] and [3] and our proposal is available in Appendix A.

\section{Results}

In this section we present and describe the characteristics of software testers derived from the study during the qualitative analyze process. Table 2 shows the characteristics sorted in quantitative descendent order starting from the characteristics mentioned by the higher number of participants:

Table 2: Characteristics of software testers

\begin{tabular}{|l|l|l|l|}
\hline $\begin{array}{l}\text { Most wanted tester } \\
\text { characteristics }\end{array}$ & Rank & $\begin{array}{l}\text { Most wanted tester } \\
\text { characteristics }\end{array}$ & Rank \\
\hline Communication skills & 7 & Diplomacy & 3 \\
\hline IT background & 6 & Need for challenge & 3 \\
\hline Need for variety & 6 & Domain knowledge & 3 \\
\hline Details oriented & 5 & Courage & 3 \\
\hline Curious & 4 & Creative & 2 \\
\hline Passion for quality & 4 & Proactive & 2 \\
\hline Patient & 4 & Structured & 2 \\
\hline Testing experience & 4 & Team player & 2 \\
\hline Achievement orientated & 3 & Mind set to find bugs & 2 \\
\hline
\end{tabular}

\section{Communication}

Good communication skills is the most cited characteristic. Participants mentioned that during the communication process the testers must be able to know how to provide the right information and how to communicate it properly to the parties involved. "The communication characteristic, both oral and by writing is very important in a team because we often give some bad news (defects or problems) and it must be communicated in a way that do not blame anyone for it." (Tester Manager, Company C

A tester is required to communicate with different project team members, managers, sometimes clients, to report defects, discuss and follow them up with the developers: "The tester needs communication skills in order to communicate with the project manager, developers and the users, and he has to make them understandable to each other" (Tester, Company C) 


\section{IT background}

Possessing an IT background refers to having an education and/or knowledge within computer science fields. Interviewees in this study held programming knowledge and experience, as valuable assets due to the expertise accumulated which can help in certain testing activities. "A development experience can help a lot. All the knowledge I gain while working as a developer, helped me in my testing work." (Tester, Company A)

An IT background was considered an important characteristic for a tester by our interviewees. The IT background proved valuable when communicating with the developers. "In order to speak to a developer and understand what they are talking about, an IT background is a very good thing”. (Tester, Company B)

\section{Need for variety}

In all participant companies the Need for variety characteristic was often cited as a positive trait due to the varying nature of tasks in which a tester could be involved. A testers who is enjoys having a variety of tasks could adapt better to the different nature responsibilities alongside a project lifecycle starting from the design phase until the maintenance stage. "The biggest factor for me is that you do different things, it's very varied and you get to see the whole picture. You can participate from the start of a project to the end doing various things, that's the biggest thing for me, the different things I can do." (Tester Manager, Company B).

\section{Details oriented}

Being a details oriented person was considered an advantageous skill for a tester among the study participants. "You have to have an eye for details... you cannot just check the normally expected results. You cannot just test the happy path scenarios”. (Tester Manager, Company B)

\section{Curious}

Some of the participant considered that being curious was an incentive for continuously improving the understanding of the product and for pursuing several testing scenarios. Curiosity was also advantageous for coming up with unusual testing scenarios. "You don't have to be afraid to click on new things and that you are going to break something. That is part of being curious." (Tester Manager, Company D) "Curiosity. You should want to learn new things because you will get new software, new projects, new teams all the time" (Tester, Company B)

\section{Passion for quality}

Some of the participants have expressed their desire to improve the quality in the products they are working on and taking pride in participating in the delivery of a high quality product. They talked about their joy in investigating and finding defects which will lead to a better product. "I do have a passion for improving the quality and finding defects... I'm happy when I find bugs. Of course, I'm also happy when things are working." (Tester, Company A)

\section{Patient}

Patience was mentioned is being an important requirement for software testers. The testers may need to work outside of regular office with little warning in advance. "you say: <<today I'll leave at 4>>, but at 3:45 they say <<you have to do this testing before you leave today $>>$, and you have to work 2 or 3 hours more. That's a part of it" (Tester Manager, Company D) 
Patience was considered critical in situations where often and recurrently the planned scheduled was discarded and new plans were proposed. "I think you have to be patient, because usually you are at the end of the cycle and your plans and timetables are going to change, a lot." (Tester, Company A)

\section{Testing experience}

The interviewees thought that testing experience and testing knowledge could improve the design skills such as writing Test Cases: "Defining the TC's is very important and you find all kind of situations where you need quite a lot of fantasy, since you have to go further than the developers go. And that has a lot to do with experience" (Tester Manager, Company A)

Experience with requirements engineering was also valued among the interviewed testing participants: "I would like them to have some competency in requirements, how should requirements be, because often they will be asked to review requirements and provide feedback." (Tester Manager, Company D)

\section{Discussion}

The answer to RQ1, "What are the characteristics perceived as important of software testers?" can be constructed by starting from the information provided in Table 2. Communication skills are seen as a valuable characteristic both from the participants of this study and from the research literature. During the communication process the testers must be able to know how to provide the right information and how to communicate it properly to the parties involved. For example, management may not be interested in the details of every defect that has been located. They are interested in the overall quality of a release and if the release can go in production. Testers have to be able to write clear defects reports and they also must be able to describe a problem while taking into consideration the type of interlocutors: "The communication characteristic, both oral and by writing is very important in a team because we often give some bad news (defects or problems) and it must be communicated in a way that do not blame anyone for it." (Tester Manager, Company C).

One contrasting results with [2] and [3] is that the three most rated characteristics, growth orientation, need for independence and low need for social interaction associated with introverts did not score high with our participants. The characteristic of being Introverted was not mentioned at all in our interviews. This seems to be one of the strong difference between a tester and a developer. If you are introverted, then spending most of your workday behind a computer screen alone, as a developer, is a good choice, but not necessarily for a tester. Testers often have to report problems and they have to be comfortable with conflict [7], which lead to a vast amount of communication on daily basis. They have to learn a new product or feature in short time and posses or develop an ability, so a certain need for variety and curiosity might prove the right incentive. A note of caution should be taken for this characteristic, the need of variety is a useful trait in a domain where you might often need to acquire new knowledge of a new topic, but a strong need for variety may clash with the 
possible monotonous nature of some testing activities and desired characteristics such as patience and tolerance to tedium which corroborate the findings of Pettichord [7].

If we compare our results with the characteristics derived from studies for Software Engineers [2] and [3], we notice that communication is not mentioned among the study's result, which leads us to the conclusion that communication skills is a specific valuable trait for a tester, hence proving an answer for our second research question: "Are there specific characteristics that separates software testers from a software engineer?".

Other characteristics which were not present in [2] and [3] are patience and curiosity. Patience is a virtue, especially among testers. They might invest a lot of effort in testing a new release and it may turn out that an incorrect version was deployed, so all their test results are worthless. During the testing process, testers might encounter opposition, aggression or debating situations which require patience. A tester might need to listen to the comments, criticism and deal with frustrated colleagues or managers on a daily basis. Software Testing can and should be seen as a journey for discovering and exploring freshly delivered software. A curious and inquisitive mind will be important to understand the why and how of products, their interactions and dependencies.

In most of the existing studies on testing domain knowledge and testing experience are ranked highly, but our participant emphasised the need for an IT background. This type of background can provide knowledge and language which will improve the testers ability to write technical reports, relate and discuss with developers.

One company valued the domain knowledge above the technical ones due to the sensitivity and complexity for the of business they were involved in. One possible explanation is that in some of the participant organizations, parts of testing are done by non-specialized software testers alongside their other activities, or as a temporary task according to the projects' needs. In these cases, the people performing the testing usually lack testing skills, experience, and training which can lead to difficulties when communicating with more technical member of the team and when reporting defects. This situation was also described in Pol's study [9], and one of the consequences is that testing is seen as a job anyone can do.

As explained by Black [8], we have to remind ourselves and be aware that different levels of testing requires different skills. Unit testing requires programming and software development skills, while specialized tester are doing functional testing. When the process reach the level of acceptance or beta testing the most fitted testers are the one with best user knowledge such as people from customer support or business analysts. One negative aspect of this practice will be to rely too much on borrowing people from support group for testing, since they might not have sufficient knowledge for testing at earlier stages.

\section{Limitations and threats to validity}

In order to avoid threats to validity presented by Robson [16] in this kind of research, we ensured observer triangulation by having the data analyzed by three researchers, while the collected data and the results of this study were compared with 
our earlier quantitative study [17], which allowed us to apply data and method triangulation.

We are aware that the low number of participants is a limitation and given the high number of variables playing an important role in the survey, the results of this study should be considered as preliminary. However, since the focus was on depth rather than breadth we still think that the participants provided a typical sample giving us a lot of input and perspectives. Since increasing the number of participants could reveal more details and strengthen the conclusion of this study, our plan is to further expand our research by inviting new companies and increase the total number of interviewees. In addition, we will enhance our target population by inviting developers and project managers to take part our study. This augmentation could provide us with valuable information on the desirable characteristics for testers as seen from the developers perspective and from a higher managerial angle.

In addition to the small sample research, other factors such as the organizational mode or the type of product might influence the characteristics which were most valued or encountered in the participant companies. Nevertheless, our qualitative analysis spanned across four companies with mixed sizes and methodologies, involved in national and international business, handling data with medium or high risk. The participants were involved in performing functional and non-functional testing, and were coming from a mixed background with some having good technical skills and an IT background, while other were poses more strength in the domain business knowledge. All the mentioned factors could give better generalizability of findings than performing interviews in just one company [15].

\section{Conclusion}

In this paper we present the results of a qualitative survey among four Norwegian companies and the characteristics which were perceived as most valuable for a software tester. We contrasted our results with exiting work on software engineers' characteristics and noticed that the most frequent cited characteristics for software engineers were not applicable to software testers. A strong emphasis was laid on communication skills and on IT background which implied programming knowledge and certain technical capabilities. Testing knowledge and domain knowledge were also valued but not as strongly as found in previous research conducted Beer and Ramler [11]. Testing experience was considered to be an advantage especially for the testers involved in domains with volatile requirements which seems to be in line with Martin, Rooksby et al. [10] earlier study.

The list of characteristics provided by our study should not be seen as exhaustive or definitive but rather as a starting point and each company should consider the type of knowledge they value at hiring and the ability to provide internal training for testing or domain knowledge for their relevant business. 


\section{Acknowledgment}

We are very grateful to the companies and their employees for their contribution to this project. Also, we would like to thank to Torgeir Dingsøyr at the Norwegian University of Science and Technology, for comments of versions of this article.

\section{References}

1. Rooksby, J., Rouncefield, M., Sommerville, I.: Testing in the Wild: The Social and Organisational Dimensions of Real World Practice. Comput. Support. Coop. Work. 18, 559-580 (2009).

2. Beecham, S., Baddoo, N., Hall, T., Robinson, H., Sharp, H.: Motivation in Software Engineering: A systematic literature review. Inf. Softw. Technol. 50, 860-878 (2008).

3. Franca, A.C.C., Gouveia, T.B., Santos, P.C.F., Santana, C.A., da Silva, F.Q.B.: Motivation in software engineering: a systematic review update. 15th Annual Conference on Evaluation \& Assessment in Software Engineering (EASE 2011). pp. 154-163. IET (2011).

4. DeMarco, T., Lister, T.: Peopleware: Productive Projects and Teams. Dorset House (1999)

5. Shah, H., Harrold, M.J., Sinha, S.: Global software testing under deadline pressure: Vendor-side experiences. Inf. Softw. Technol. 56, 6-19 (2014).

6. Cohen, C.F., Birkin, S.J., Garfield, M.J., Webb, H.W.: Managing conflict in software testing. Commun. ACM. 47, 76-81 (2004).

7. Pettichord, B.: Testers and developers think differently: Understanding and utilizing the diverse traits of key players on your team. Softw. Test. Qual. Eng. 2, 42-45 (2000).

8. Black, R.: Managing the Testing Process - Practical Tools and Techniques for Managing Hardware and Software Testing. Wiley Publishing Inc., New York, New York, USA (2002).

9. Pol, M., Teunissen, R., Veenendaal, E. van: Software Testing: A Guide to the TMap Approach. Pearson Education (2002).

10. Martin, D., Rooksby, J., Rouncefield, M., Sommerville, I.: “Good” Organisational Reasons for "Bad” Software Testing: An Ethnographic Study of Testing in a Small Software Company. 29th International Conference on Software Engineering (ICSE’07). pp. 602-611. IEEE (2007).

11. Beer, A., Ramler, R.: The Role of Experience in Software Testing Practice. 2008 34th Euromicro Conference Software Engineering and Advanced Applications. pp. 258-265. IEEE (2008).

12. Iivonen, J., Mantyla, M., Itkonen, J.: Characteristics of High Performing Testers - A Case Study. Proceedings of the 2010 ACM-IEEE international symposium on empirical software engineering and measurement. p. 1. ACM (2010).

13. Taipale, O., Smolander, K.: Improving software testing by observing practice. Proc. 2006 ACM/IEEE Int. Symp. Int. Symp. Empir. Softw. Eng. - ISESE ’06. 262 (2006).

14. Riungu, L.M., Taipale, O., Smolander, K.: Software Testing as an Online Service: Observations from Practice. 2010 Third Int. Conf. Softw. Testing, Verif. Valid. Work. 418-423 (2010). 
15. Myers, M.D., Newman, M.: The qualitative interview in IS research: Examining the craft. Inf. Organ. 17, 2-26 (2007).

16. Robson, C.: Real World Research. 2nd. Ed. Blackwell Publ. Malden. (2002).

17. Deak, A., Stalhane, T.: Organization of Testing Activities in Norwegian Software Companies. 2013 IEEE Sixth International Conference on Software Testing, Verification and Validation Workshops. pp. 102-107. IEEE (2013).

\section{Appendix}

Characteristics for software engineers

\begin{tabular}{|l|l|l|}
\hline $\begin{array}{l}\text { Proposing } \\
\text { study }\end{array}$ & Software Engineer characteristics & Frequency \\
\hline \multirow{5}{*}{ Beecham 01 } & Growth orientated & 9 \\
\cline { 2 - 3 } & Introverted & 7 \\
\cline { 2 - 3 } & Autonomous & 7 \\
\cline { 2 - 3 } & Need for stability & 5 \\
\cline { 2 - 3 } & Technically competent & 5 \\
\cline { 2 - 3 } & Need to be sociable & 5 \\
\cline { 2 - 3 } & Achievement orientated & 4 \\
\cline { 2 - 3 } & Need for competent supervising & 4 \\
\cline { 2 - 3 } & Need for variety & 4 \\
\cline { 2 - 3 } & Need for challenge & 4 \\
\cline { 2 - 3 } & Need to make a contribution & 3 \\
\cline { 2 - 3 } & Need for feedback & 2 \\
\cline { 2 - 3 } & Marketable & 2 \\
\cline { 2 - 3 } & Creative & 2 \\
\cline { 2 - 3 } & Need for involvement in personal goal setting & 1 \\
\cline { 2 - 3 } & Need for Geographic stability & 2 \\
\hline \multirow{5}{*}{ Our study } & Competent in Management & 1 \\
\cline { 2 - 3 } & Flexible / Team worker (easy to work with) & 2 \\
\cline { 2 - 3 } & Have fear of punishment & 2 \\
\hline & Communication & \\
\hline
\end{tabular}

